\title{
Development of alternative versions of the Logical Memory subtest of the WMS-R for use in Brazil
}

\author{
Silvia Adriana Prado Bolognani ${ }^{1}$, Monica Carolina Miranda ${ }^{1,2}$, Marjorie Martins², Patricia Rzezak³, \\ Orlando Francisco Amodeo Bueno ${ }^{1,2}$, Candida Helena Pires de Camargo4, Sabine Pompeia ${ }^{2}$
}

\begin{abstract}
The logical memory test of the Wechsler Memory Scale is one of the most frequently used standardized tests for assessing verbal memory and consists of two separate short stories each containing 25 idea units. Problems with practice effects arise with re-testing a patient, as these stories may be remembered from previous assessments. Therefore, alternative versions of the test stimuli should be developed to minimize learning effects when repeated testing is required for longitudinal evaluations of patients. Objective: To present three alternative stories for each of the original stories frequently used in Brazil (Ana Soares and Roberto Mota) and to show their similarity in terms of content, structure and linguistic characteristics. Methods: The alternative stories were developed according to the following criteria: overall structure or thematic content (presentation of the character, conflict, aggravation or complements and resolution); specific structure (sex of the character, location and occupation, details of what happened); formal structure (number of words, characters, verbs and nouns); and readability. Results: The alternative stories and scoring criteria are presented in comparison to the original WMS stories (Brazilian version). Conclusion: The alternative stories presented here correspond well thematically and structurally to the Brazilian versions of the original stories.
\end{abstract}

Key words: logical memory, episodic memory, prose recall, memory test, stories.

\section{DESENVOLVIMENTO DE VERSÕES ALTERNATIVAS DO SUBTESTE MEMÓRIA LÓGICA DA WMS-R PARA USO NO BRASIL}

RESUMO. 0 teste Memória Lógica da Escala Wechsler de Memória é uma das tarefas mais empregadas de avaliação de memória e é composto de duas histórias sobre diferentes personagens que contém 25 itens. A repetição destas histórias para a mesma pessoa em diferentes ocasiões leva a uma melhora nos escores por efeito de familiaridade com seu conteúdo. Portanto, versões alternativas deste teste devem ser construídas de modo a permitir que o teste seja repetido em situações como a avaliação longitudinal de pacientes. Objetivo: Apresentar 3 histórias alternativas a cada uma das versões das histórias originais mais empregadas no Brasil (Ana Soares e Roberto Mota). Métodos: As histórias alternativas foram construídas obedecendo aos seguintes critérios: estrutura geral ou conteúdo temático (apresentação do personagem; conflito; agravante ou complemento; resolução); estrutura específica (sexo do personagem; localidade e ocupação, detalhes da ocorrência); estrutura formal (número de palavras, caracteres, verbos e substantivos); e legibilidade. Resultados: São apresentadas as histórias alternativas e critérios de correção em comparação às versões das histórias originais. Conclusão: as histórias alternativas apresentadas tem correspondência temática e estrutural com as versões brasileiras do teste original. Palavras-chave: memória lógica, memória episódica, recordação, teste de memória, histórias.

\section{INTRODUCTION}

The Logical Memory task from the of the most frequently used tests for assessing episodic memory. ${ }^{2}$ This test is composed of two stories which include 25 units or ideas each, and are verbally presented by the examiner: one about a woman (presented first), and the other about a man.

Immediately after each story is presented (immediate recall), the test subject is asked to repeat what they remember as accurately

\footnotetext{
This study was conducted at the Departamento e Psicobiologia, Universidade Federal de São Paulo.
}

${ }^{1}$ Centro Paulista de Neuropsicologia, Associação Fundo de Incentivo à Pesquisa. ${ }^{2}$ Departamento de Psicobiologia, Universidade Federal de São Paulo. ${ }^{3}$ Lim-21, Instituto de Psiquiatria, Universidade de São Paulo. ${ }^{4}$ nnstituto de Psiquiatria, Universidade de São Paulo.

Monica Miranda. Rua Napoleão de Barros, 925 - 04024-002 São Paulo SP - Brasil. E-mail: mirandambr@yahoo.com.br

Disclosure: The authors report no conflicts of interest.

Received March 16, 2015. Accepted in final form June 05, 2015. 
as possible, without being given any clues (free recall). ${ }^{1}$ Thirty minutes later, subjects are asked to recall the story again (delayed recall). For both immediate and delayed recall, all correct items are summed giving a maximum score of 25 for each story. The WMS is currently in its $4^{\text {th }}$ edition, ${ }^{3}$ and since its previous version, ${ }^{4}$ the logical memory stories have gained an additional form of scoring. The 25-item scoring system was maintained (which counts specific units of the story), and a second scoring criteria was established, considering the recall of general topics (gist). ${ }^{5}$ For example, the original scoring system credits points for the recall of the character's exact name and surname (one point each); the additional scoring system now also grants a point if the subject recalls that the story was about a woman, even if her name is not remembered.

Because of its great value in memory assessment, the logical memory test is often used when re-testing is needed in clinical practice and longitudinal research. Changes in memory performance are often assessed after interventions such as surgeries, ${ }^{6}$ evolution of psychiatric disorders, ${ }^{7}$ as well as several types of therapeutic and pharmacological interventions. ${ }^{8,9}$ These studies involve the assessment of the same subjects on two or more separate occasions.

The logical memory test is known to be susceptible to practice effects or training, i.e., the repeated use of the same story increases the possibility that it is remembered on subsequent assessments (stimulus learning). ${ }^{2,10}$ Thus, there is an urgent need for alternative stories. However, few studies have developed alternative stimuli. This is the first step in proposing alternative versions of cognitive tasks, the second being the study of psychometric similarity between the versions in healthy participants and clinical populations. Various studies have suggested means of constructing alternative stories to those in the WMS as discussed below. In this study, the criteria from these scientific publications were used to construct the alternative versions of the task in Portuguese.

Morris, Kunka and Rossini ${ }^{11}$ presented an alternate version for each of the WMS-R stories that had equivalent characteristics in terms of their items (units of ideas), sentences, words per sentence, syllables per word, readability and subjective attributes (such as affective tone). In many clinical and experimental settings, however, more than one alternative story for each original may be required. Sullivan ${ }^{2}$ proposed six stories following similar criteria. ${ }^{11}$ The author then analyzed the comparability of stories in terms of number of words, characters, sentences and readability, as well as the performance of normal subjects in terms of immediate recall of these stories after oral presentation. Though these stories are generally similar, there were some differences between them, probably because the original stories of the WMS-R test are not equivalent in the parameters studied, ${ }^{2}$ and also differ in terms of ease of memorization. ${ }^{12}$

Two other publications have proposed alternative stories, applying similar methods to those adopted by the studies mentioned above. ${ }^{2,11}$ Schnabe ${ }^{10}$ published two alternative versions of the WMS-IV stories that were similar in structure, narrative, and readability. Moreover, he tested the compatibility of the WMS stories and the newly developed stories empirically in three large populations, comprising patients with depression, head injury, and a control group of healthy individuals. He demonstrated that performance regarding the original and the newly developed stories was interchangeable for all groups, both for immediate and delayed recall. A further study shows three short stories similar to each other, but these were not equivalent to the stories of the WMS. ${ }^{13}$

The use of the logical memory test in Brazil poses several major difficulties. To date, the test has not been published in Portuguese for any of the WMS versions. A free translation / adaptation of the pair of stories of the WMS-R (Ana Soares and Roberto Mota) was done for clinical and research purposes at one of the major neuropsychology centers in Brazil by Camargo et al., ${ }^{14}$ but the stories in full and the scoring criteria were not published. One article carries one of the two stories in Portuguese (Ana Soares), but no scoring criteria were provided..$^{15}$ These versions have been shared privately among memory specialists for years and are probably the most common prose recall tests used by Brazilian professionals in research and clinical work. Hence, there is no publication that includes both these adapted stories and their scoring criteria, nor scientific work on standardization or systematic adaptation of this instrument for use in Brazil.

There are stories for memory testing created in Portuguese from Brazil, but these are not equivalent to the any of the versions of the WMS, since they are shorter and have a very different thematic structure. ${ }^{16,17}$ Given that many Brazilian professionals have been using the logical memory test and may need to re-test patients, it would be useful if the alternative stories in Portuguese were constructed so as to be equivalent to those adapted by Camargo et al. ${ }^{14}$ from the stories of the WMS-R.

The aim of this study was to develop a set of alternative logical memory stories to those frequently used in 
Brazil (Ana Soares and Roberto Mota) in terms of their content, structure and linguistic characteristics. The development of the material was based on parameters proposed by previous authors, ${ }^{2,10,11}$ and also took into account the thematic structure of the original stories. The material presented here, however, does not show the psychometric equivalence of the stories, which was tested later. ${ }^{18}$

\section{METHODS}

Three alternative stories for each of the stories in Portuguese (Ana Soares and Roberto Mota) ${ }^{14}$ were proposed by the authors following the criteria below, based on previous studies. ${ }^{2,10,11}$

1. General structure of the plot: each story involved the presentation of character, a conflict, an aggravation / complements to the main plot, and a resolution. The alternative stories to that of Ana Soares involved female characters whereas those of the Roberto Mota stories had male characters.

2. Specific elements: all stories presented the characters with a first name, surname, occupation and place of origin; the stories that were alternatives for the female story had numerals and the male ones had closure statements with a specific word that can be considered slightly out of the context that can be scored even if recalled alone (equivalent to the word 'shark' in the Roberto Mota story).

3. Grammatical structure and details: all stories were equivalent regarding the grammatical classes of words, number of words, letters, verbs and nouns per idea item and in total;

4. Units of general topics (gist): units that summarize the essential ideas of the excerpts of the story were considered, allowing little specific recollection to be granted a point on the secondary scoring system as mentioned in the introduction section. The units of general themes of the female stories were guided directly by those proposed by the WMS-III, which maintains the story equivalent to that of Ana Soares, with a total of 7 themes. The male stories had these units freely defined by the present authors, since the story that led to the Roberto Mota version was not maintained in the most recent versions of the test in its original English version; this secondary scoring has a total of 8 units for the stories of the male characters.

5. Readability: reading complexity was calculated with the Flesch Reading Ease Scores adapted for local use in Portuguese. Scores between 75 and 100 denote very easy to read, from 50 to 75 easy, 25 to 50 difficult and below 25 very difficult to read. ${ }^{19}$
Following the creation of the alternative stories, they were presented to a group of experts from the Psychology Department of the Universidade Federal de Sao Paulo, including memory researchers from different areas of Brazil (various sessions including more than 15 experts in each). This group of experts proposed changes that were necessary to make the stories easy to understand and equivalent to each other. The authors then determined the scoring criteria, which were subsequently evaluated by the group of memory experts. The final selection of acceptable responses was defined by consensus among the authors. The Appendix shows the scoring criteria for each story.

\section{RESULTS}

The original stories in Portuguese $\mathrm{e}^{14}$, and their organization according to thematic units, can be seen in Table 1. The new alternative stories are presented in Table 2 , which also shows the equivalence to the original stories according to various parameters such as: thematic content, structure (sex of the character, their origin and profession, what happened and the resolution of the problem), grammatical details, as well as readability of stories, all of which were scored as easy to read according to the Flesch Readability Ease Scores (between 50 and 75).${ }^{19}$ The Appendix shows the scoring criteria with the acceptable answers, both for the main score, which requires greater accuracy in recall, as well as for the secondary scoring, which evaluates the ability to remember general themes (gist). Examples of incorrect responses are also presented.

\section{DISCUSSION}

The logical memory test is one of the most used tasks for assessing episodic memory in both clinical and research settings. ${ }^{2}$ In many cases, reassessment of patients is needed. ${ }^{6-9}$ The repeated use of this kind of test leads to learning effects due to many factors, ${ }^{10}$ including recall of the story content when the same story is told more than once. The use of different stories that have similar characteristics is one of the methods used to improve the validity of repeated assessments. ${ }^{10}$ The present study presents three alternative stories for each of the freely translated stories from the WMS-R, as these are frequently used in Brazil despite the lack of an official translation and of validation in the Brazilian population. The creation of the alternative stories was based on criteria proposed by authors who developed English versions of the task (narrative structure, emotional content, readability, number of words, etc.). ${ }^{2,10,11}$ The comparability of stories in terms of the plot, structure and 
Table 1. Stories translated and adapted by Camargo et al. ${ }^{11}$ for use in Brazil and thematic structure (plot structure).

\begin{tabular}{|c|c|c|c|}
\hline \multicolumn{2}{|c|}{ Female character (Ana Soares) } & \multicolumn{2}{|c|}{ Male character (Roberto Mota) } \\
\hline Structure & Story items & Structure & Story items \\
\hline \multirow{8}{*}{$\begin{array}{l}\text { Character } \\
\text { introduction }\end{array}$} & Ana & \multirow{11}{*}{$\begin{array}{l}\text { Character } \\
\text { introduction }\end{array}$} & Roberto \\
\hline & Soares & & Mota \\
\hline & do Sul & & estava dirigindo \\
\hline & do Paraná & & um caminhão \\
\hline & empregada & & Mercedes \\
\hline & como faxineira & & numa rodovia \\
\hline & em um prédio & & à noite \\
\hline & de escritórios & & no Vale \\
\hline \multirow[t]{8}{*}{ Problem } & relatou & & do Paraíba \\
\hline & na delegacia & & levando ovos \\
\hline & de polícia & & para Taubaté \\
\hline & que tinha sido assaltada & \multirow[t]{5}{*}{ Problem } & quando 0 eixo \\
\hline & na Rua do Estado & & quebrou. \\
\hline & na noite anterior & & 0 caminhão derrapou \\
\hline & e roubada & & caindo numa valeta \\
\hline & em 150 Reais. & & fora da estrada. \\
\hline \multirow[t]{5}{*}{ Aggravation } & Ela disse que tinha 4 & \multirow[t]{5}{*}{ Aggravation } & Ele foi jogado \\
\hline & filhinhos & & contra o painel \\
\hline & 0 aluguel não tinha sido pago & & e se assustou muito. \\
\hline & e eles não comiam & & Não havia trânsito \\
\hline & há dois dias. & & e ele duvidou que pudesse ser socorrido. \\
\hline \multirow[t]{4}{*}{ Resolution } & Os policiais & \multirow[t]{4}{*}{ Resolution } & Naquele instante, seu radio amador \\
\hline & tocados pela historia da mulher & & tocou. \\
\hline & fizeram uma coleta & & Ele respondeu imediatamente: \\
\hline & para ela. & & "Aqui fala tubarão". \\
\hline
\end{tabular}

the linguistic features were presented here, and detailed information on their psychometric equivalence can be found in the study of Martins et al., ${ }^{18}$ which shows the performance of a non-clinical sample on the immediate and delayed recall of the alternative stories.

Besides the alternative stories that are similar to the Portuguese version of the WMS-R, the material presented here also includes the additional scoring criteria (gist or general themes) introduced in later versions of the WMS. ${ }^{3,4}$ This additional score has important clinical and experimental value, since it helps differentiate levels of impairment and allows a more detailed investigation of memory not only as an all-or-nothing phenomenon, but rather as the storage of stimuli at different levels of specificity. ${ }^{20,21}$

Finally, it is important to acknowledge several limitations of the present study. First of all, there are no available validated versions of the WMS stories in Portuguese for use in Brazil. Hence, the stimuli used as "models" for this study were those proposed by $\mathrm{Ca}$ margo et al. ${ }^{14}$ which have been extensively used by neuropsychologists in Brazil despite not having been published in a scientific journal. The second point refers to the circumscribed aim of the present article, describing only the development of the stories, without testing their validity for clinical populations. This was necessary to show that the alternative versions were created in a similar fashion to those available in English and published in the international literature. Their equivalence to the model stories, although not presented here, was reported elsewhere. ${ }^{18} \mathrm{~A}$ third issue that should be pointed out is the absence of Brazilian norms for the 
Table 2. Stories translated and adapted by Camargo et al. ${ }^{11}$ for use in Brazil (original) according to plot structure, alternative stories with female (a) and male (b) characters and proposed parameters of comparability between versions.

\begin{tabular}{|c|c|c|c|c|}
\hline \multicolumn{5}{|c|}{ a. Female characters (F) } \\
\hline Plot structure & Original story (f) & Alternative story (F1) & Alternative story (F2) & Alternative story (F3) \\
\hline \multirow{8}{*}{$\begin{array}{l}\text { Character } \\
\text { introduction }\end{array}$} & Ana & Maria & Suzana & Regina \\
\hline & Soares & da Conceição, & Borges & Cardoso, \\
\hline & do Sul & do sertão & da periferia & do interior \\
\hline & do Paraná, & do Ceará, & de Salvador, & de Minas Gerais, \\
\hline & empregada & trabalhava & estudante & instrutora \\
\hline & como faxineira & de lavadeira & de direito & de tênis \\
\hline & em um prédio & para o prefeito & na Universidade & no clube \\
\hline & de escritórios, & da cidade. & Federal, & da prefeitura, \\
\hline \multirow[t]{9}{*}{ Problem } & relatou, & Numa manhã, & Reclamou & estava brava \\
\hline & na delegacia & encontrou, & no escritório & com os funcionários \\
\hline & de polícia & no bolso & da diretoria & da limpeza. \\
\hline & que tinha sido assaltada & de uma calça suja, & que tinham sumido, & No dia anterior \\
\hline & na Rua do Estado & uma caixinha bonita & de cima de sua mesa, & eles ficaram brincando \\
\hline & na noite anterior & contendo um anel & na tarde anterior, & com as raquetes dela e \\
\hline & e roubada & de ouro & os livros & depois não guardaram de volta \\
\hline & em 150 Reais. & e uma nota fiscal & que ela tinha comprado. & no lugar certo \\
\hline & & no valor de 2.000 Reais. & & \\
\hline \multirow[t]{5}{*}{ Aggravation } & Ela disse que tinha 4 & A joia & Ela disse que ia ter 3 & Ela chamou duas \\
\hline & filhinhos, & escapou de suas mãos & provas, & de suas colegas \\
\hline & 0 aluguel não tinha sido pago & e caiu no ralo. & que ainda não tinha estudado, & para dar "uma lição" neles \\
\hline & e eles não comiam & & e que os exames aconteceriam & no domingo. \\
\hline & há dois dias. & & dali a 2 dias. & \\
\hline \multirow[t]{5}{*}{ Resolution } & Os policiais & Ela pegou um galho & A diretora & Elas esconderam \\
\hline & tocados pela historia da mulher & de árvore & preocupada c/ a situação da menina & os uniformes deles \\
\hline & fizeram uma coleta & para tentar fisgar 0 anel. & emprestou livros & e só devolveram \\
\hline & para ela. & Depois de 30 minutos & para ela. & quando pediram desculpas \\
\hline & & finalmente conseguiu recuperá-lo. & & para ela. \\
\hline \multicolumn{5}{|c|}{ Comparability criteria } \\
\hline No. of items & 25 & 25 & 25 & 25 \\
\hline Flesch reading ease & 55.7 & 71.2 & 52.6 & 62.9 \\
\hline No. of words & 66 & 69 & 67 & 66 \\
\hline No. of characters & 323 & 318 & 335 & 334 \\
\hline No. of verbs & 7 & 7 & 7 & 7 \\
\hline No. of nouns & 15 & 19 & 17 & 17 \\
\hline
\end{tabular}


Table 2. Continuation.

\begin{tabular}{|c|c|c|c|c|}
\hline \multicolumn{5}{|c|}{ b. Male characters (M) } \\
\hline Plot structure & Original story (m) & Alternative story (m1) & Alternative story (m2) & Alternative story (m3) \\
\hline \multirow{11}{*}{$\begin{array}{l}\text { Character } \\
\text { introduction }\end{array}$} & Roberto & Luis & José & Alberto \\
\hline & Mota & Marques & Oliveira & Lemos \\
\hline & estava dirigindo & adorava & jogava & do interior \\
\hline & um caminhão & escutar música & futebol & de Goiás \\
\hline & Mercedes & clássica. & de salão & plantava \\
\hline & numa rodovia & Seu primeiro & todo domingo & verduras e \\
\hline & à noite & filho & na quadra & legumes \\
\hline & no Vale & nasceu na maternidade & da cooperativa & no seu sítio. \\
\hline & do Paraíba & Santa Luzia, & agrícola. & Toda semana, \\
\hline & levando ovos & & Estava treinando com o time & ele ia vender os produtos \\
\hline & para Taubaté & & para um campeonato & em Itapira \\
\hline \multirow[t]{8}{*}{ Problem } & quando 0 eixo & e chorava muito. & quando sua chuteira & 0 verão \\
\hline & quebrou. & Ele percebeu & desamarrou. & estava escaldante, \\
\hline & 0 caminhão derrapou & que 0 silêncio acabara & 0 jogador tropeçou & 0 ar estava seco, \\
\hline & caindo numa valeta & e ele não poderia mais ouvir & caindo de costas & e não havia previsão de chuva \\
\hline & fora da estrada. & seus CDs. & fora do campo. & para aquela região. \\
\hline & Ele foi jogado & Quando a criança & & \\
\hline & contra o painel & completou 8 meses & & \\
\hline & e se assustou muito. & & & \\
\hline \multirow[t]{3}{*}{ Aggravation } & Não havia trânsito & ele estava desesperado, & Ele tentou levantar, & Então ele teve medo \\
\hline & $\begin{array}{l}\text { e ele duvidou que pudesse ser } \\
\text { socorrido. }\end{array}$ & pois 0 aparelho de som & mas sua perna & que a colheita \\
\hline & & $\begin{array}{l}\text { somente tocava musiquinhas } \\
\text { infantis. }\end{array}$ & doía muito. & fosse prejudicada. \\
\hline \multirow[t]{6}{*}{ Resolution } & $\begin{array}{l}\text { Naquele instante, seu radio } \\
\text { amador }\end{array}$ & Então, ele trocou o CD & Foi carregado de maca & Como o tempo não mudaria, \\
\hline & tocou. & e colocou uma sinfonia & e levado para o vestiário. & $\begin{array}{l}\text { ele resolveu tomar uma } \\
\text { providência: }\end{array}$ \\
\hline & Ele respondeu imediatamente: & de Beethoven. & 0 médico & Contratou \\
\hline & "Aqui fala Tubarão". & Para sua surpresa & o examinou & caminhões-pipa \\
\hline & & a criança se acalmou. & e viu que não havia fratura. & para regar a plantação \\
\hline & & E a casa virou o Paraíso & Ele ficou feliz como uma Criança & $\begin{array}{l}\text { que ficou verde como } \\
\text { Esmeralda }\end{array}$ \\
\hline \multicolumn{5}{|c|}{ Comparability criteria } \\
\hline No. of items & 25 & 25 & 25 & 25 \\
\hline Flesch reading ease & 60,7 & 65,6 & 66,1 & 58,1 \\
\hline No. of words & 64 & 73 & 67 & 69 \\
\hline No. of characters & 384 & 364 & 330 & 353 \\
\hline No. of verbs & 11 & 11 & 11 & 9 \\
\hline No. of nouns & 18 & 19 & 20 & 20 \\
\hline
\end{tabular}


test. For research purposes, it is recommended that matching control groups be used. However, until appropriate validation studies and norms for the WMS logical memory test are published in Brazil, the alternative stories and detailed correction criteria presented here may be used. These items may also represent a first step toward the standardization and production of norms of this task for the Brazilian population. Lastly, it must be born in mind that even with the use of alternative versions, practice effects can occur as a result of decreases in test anxiety due to familiarity with the test setting, testing procedure, and/or experimenter or clinician, as well as due to procedural learning and development of test strategies. ${ }^{10}$ The impact of these factors warrant attention in future studies employing the use of more than one story in the same individual, and may differ depending on the cognitive status of the patient.
Acknowledgments. Aos participantes do grupo de estudos de memória do Departamento de Psicobiologia da UNIFESP por suas contribuições no desenvolvimento deste trabalho e aos seguintes órgão financiadores: AFIP, CNPq e CAPES.

Author contributions. Silvia Bolognani. Study conception, methodology, data presentation, discussion and writing the initial draft and revision. Monica Miranda. Study conception, methodology, discussion, critical review. MarjorieMartins. Methodology, data presentation, discussion. Patricia Rzezak. Study conception, methodology, data presentation, discussion. Orlando Bueno. Study conception, methodology, discussion, critical review, supervision. Sabine Pompeia. Study conception, methodology, critical review, writing the revision.

\section{REFERENCES}

1. Wechsler D. Manual for the Wechsler Memory Scale - Revised. San Antonio: TX: The Psychological Corporation; 1987.

2. Sullivan K. Alternate forms of prose passages for the assessment of auditory-verbal memory. Arch Clin Neuropsychol 2005;20:745-753.

3. Wechsler D. Wechsler Memory Scale - Fourth Edition (WMS-IV) Technical and interpretative manual. San Antonio, TX: Pearson; 2009.

4. Wechsler D. Wechsler Memory Scale - Third Edition (WMS-III). San Antonio, TX: The Psychological Corporation; 1997.

5. Anand R, Chapman SB, Rackley A, Keebler M, Zientz J, Hart J Jr. Gist reasoning training in cognitively normal seniors. Int $\mathrm{J}$ Geriatr Psychiatry 2010;26:961-968.

6. Harvey DJ, Naugle RI, Magleby J, et al. Relationship between presurgical memory performance on the Wechsler Memory Scale-III and memory change following temporal resection for treatment of intractable epilepsy. Epilepsy Behav 2008;13,372-375.

7. Maeshima $\mathrm{H}$, Baba $\mathrm{H}$, Nakano $\mathrm{Y}$, et al. Time course for memory dysfunction in early-life and late-life major depression: A longitudinal study from the Juntendo University mood disorder project. J Affect Disord 2008;151:66-70.

8. Vythilingam M, Vermetten E, Anderson GM, et al. Hippocampal Volume, Memory, and Cortisol Status in Major Depressive Disorder: Effects of Treatment. Biol Psychiatry 2004;56:101-112.

9. Nikendei C, Funiok C, Pfüller U, Zastrow A, Aschenbrenner S, Weisbrod M, Herzog W, Friederich HC. Memory performance in acute and weight-restored anorexia nervosa patients. Psychol Med 2011;41:829838.

10. Schnabel R. Overcoming the challenge of re-assessing logical memory. Clin Neuropsychol 2012;26:102-115.

11. Morris J, Kunka J M, Rossini ED. Development of alternate paragraphs for the Logical Memory subtest of the Wechsler Memory Scale-Revised. Clin Neuropsychol 1997;11:370-374.

12. Henry GK, Adama RL, Buck P, Buchanan WL, Altepeter TA. The Amer- ican Liner New York and Anna Thompson: an investigation of interference effects on the Wechsler Memory Scale. J Clin Exp Neuropsychol 1990;12:502-506

13. Cunje A, Molloy D, Standish TI, Lewis DL. Alternate forms of logical memory and verbal fluency tasks for repeated testing in early cognitive changes. Int Psychogeriat 2007;19:65-75.

14. Camargo CHP, Bezerro A, Gouveia F, Santos AR, Lemos J. Teste de memória verbal: auxílio para seleção cirúrgica na epilepsia. XIX Reunião da Liga Brasileira de Epilepsia (XIV Reunião da Sociedade de Neurofisiologia Clínica); 1994; Campinas - SP.

15. Noffs MH, Magila MC, Santos AR, Marques C. Avaliação neuropsicológica de pessoas com epilepsia: visão crítica dos testes empregados na população brasileira. Neurociências 2002;10:83-93.

16. Correa DD, Gorenstein C. Bateria de testes de memória. Parte 1: critérios de elaboração e avaliação. Arq Bras Psicol 1988a;38:24-35.

17. Correa DD, Gorenstein C. Bateria de testes de memória. Parte 2: critérios de elaboração e avaliação. Arq Bras Psicol 1988b;40:42-53.

18. Martins MR, Pompéia S, Bolognani SAP, Miranda MC, Bueno OFA. Versões alternativas do subteste Memória Lógica da WMS-R: análise de desempenho de uma amostra saudável da cidade de São Paulo. Psicol Refl Crít 2015;(28)3.

19. Martins TBF, Ghiraldelo CM, Nunes MGV, Oliveira Jr ON. Readability Formulas Applied to Textbooks in Brazilian Portuguese. Instituto de Ciências Matemáticas de São Carlos-USP, PDF Version. São Carlos, Brazil. 1996 [cited 9 jul 2013]. Available from: http://www.nilc.icmc.usp. br/nilc/download/Reltec28.pdf

20. Budson AE, Todman RW, Schacter DL. Gist Memory in Alzheimer's Disease: Evidence From Categorized Pictures. Neuropsychology 2006; 20:113-122.

21. Oyarzu JP, Packard PA. Stress-Induced Gist-Based Memory Processing: A Possible Explanation for Overgeneralization of Fear in Posttraumatic Stress Disorder. J Neurosci 2012;32:9771-9772. 


\section{APPENDIX}

Alternative stories and complete scoring criteria for items and general themes (gist). The tables below may be used as scoring sheets for each story.

\begin{tabular}{|c|c|c|c|c|}
\hline \multicolumn{5}{|c|}{ F1. MARIA DA CONCEIÇÃO } \\
\hline História & $\begin{array}{l}\text { Pontuação ( } 0 \text { ou } 1 \text { ) } \\
\text { Sinônimos ou ideias } \\
\text { completas }\end{array}$ & $\begin{array}{l}\text { Pontuação (0 ou } 1 \text { ) } \\
\text { Temas ou ideias } \\
\text { gerais (gist) }\end{array}$ & $\begin{array}{l}\text { Sinônimos } \\
\text { (1 ponto) }\end{array}$ & Não pontuar \\
\hline Maria & & & Maria & Outro nome \\
\hline da Conceição & & & Conceição & Outro sobrenome \\
\hline do sertão & & & Sertão, Interior & Outro local \\
\hline do Ceará & & & Ceará, Cearense & Outro Estado \\
\hline TEMA GERAL (GIST) & & & $\begin{array}{l}\text { INDICAÇÃO DE QUE } 0 \text { PERSONAGEM PRINCIPAL } \\
\text { ERA MULHER }\end{array}$ & \\
\hline trabalhava & & & Trabalhava, Estava empregada & \\
\hline de lavadeira & & & Lavadeira, Lavava roupa & $\begin{array}{l}\text { Faxineira, Costureira, Doméstica, } \\
\text { Empregada }\end{array}$ \\
\hline para o prefeito & & & Prefeito, Casa do Prefeito & $\begin{array}{l}\text { Chefe, Coronel, Deputado, Patrão, } \\
\text { Presidente }\end{array}$ \\
\hline da cidade. & & & Cidade & Outro lugar \\
\hline TEMA GERAL (GIST) & & & $\begin{array}{l}\text { INDICAÇÃO DE QUE O PERSONAGEM TINHA UM } \\
\text { TRABALHO }\end{array}$ & \\
\hline Numa manhã & & & Numa manhã, De manhã & Certa vez, Certo dia, Um dia \\
\hline encontrou & & & Encontrou, Achou & Comprou, Ganhou \\
\hline no bolso & & & Bolso & Qualquer outro lugar \\
\hline de uma calça suja & & & Calça suja, Roupa suja & Calça velha, Casaco, Paletó \\
\hline uma caixinha bonita & & & Caixinha, Caixa & Coisa, Carteira \\
\hline contendo um anel & & & Anel, Joia, Brilhante, Aliança, $\left({ }^{\star}\right)$ & Cordão, Relógio \\
\hline TEMA GERAL (GIST) & & & $\begin{array}{l}\text { INDICAÇÃO DE QUE O PERSONAGEM ACHOU } \\
\text { UM PERTENCE ALHEIO }\end{array}$ & \\
\hline de ouro & & & Ouro, Brilhante, Dourado $\left({ }^{*}\right)$ & Valioso \\
\hline e uma nota fiscal & & & Nota fiscal, Recibo & Cheque, Valor em dinheiro, Folha \\
\hline no valor de 2.000 Reais. & & & 2.000 Reais & $\begin{array}{l}\text { 12, } 100 \text { reais, Dinheiro, } 5 \text { mil } \\
\text { cruzados, } 1 \text { real }\end{array}$ \\
\hline TEMA GERAL (GIST) & & & $\begin{array}{l}\text { INDICAÇÃO DE QUE } 0 \text { OBJETO ERA CARO OU } \\
\text { VALIOSO }\end{array}$ & \\
\hline A joia & & & Joia, o Brilhante, Anel (*) & A caixa, Caixinha \\
\hline escapou de suas mãos & & & Escapou, Caiu, Deixou Cair, Derrubou & Perdeu \\
\hline e caiu no ralo. & & & Ralo & $\begin{array}{l}\text { Buraco, No rio, Lago, No chão, } \\
\text { Bueiro, Cano }\end{array}$ \\
\hline TEMA GERAL (GIST) & & & $\begin{array}{l}\text { INDICAÇÃO DE QUE } 0 \text { OBJETO SE PERDEU, OU } \\
\text { QUE CAIU }\end{array}$ & \\
\hline Ela pegou um galho & & & $\begin{array}{l}\text { Galho, Pedaço de pau, Graveto, Vara, Vareta, } \\
\text { Varinha }\end{array}$ & $\begin{array}{l}\text { Pegou um palito, Garfo, Linha } \\
\text { e anzol }\end{array}$ \\
\hline de árvore & & & De árvore & \\
\hline para tentar fisgar 0 anel. & & & Tentar fisgar, Pescar, Recuperar, Pegar & Tentou retirá-lo \\
\hline Depois de 30 minutos & & & 30 minutos & $\begin{array}{l}\text { Algum tempo, } 2 \text { minutos, } 2 \text { horas, } \\
\text { Depois de muito esforço }\end{array}$ \\
\hline TEMA GERAL (GIST) & & & $\begin{array}{l}\text { INDICAÇÃO DE QUE HOUVE ESFORÇO } \\
\text { TENTANDO RECUPERAR O OBJETO }\end{array}$ & \\
\hline $\begin{array}{l}\text { finalmente conseguiu } \\
\text { recuperá-lo. }\end{array}$ & & & $\begin{array}{l}\text { Recuperar, Retirar, Tirar, Conseguiu, Pegar, } \\
\text { Expressão da ideia de ter êxito. }\end{array}$ & Achou novamente \\
\hline TEMA GERAL (GIST) & & & $\begin{array}{l}\text { INDICAÇÃO DE QUE } 0 \text { OBJETO FOI } \\
\text { RECUPERADO }\end{array}$ & \\
\hline Pontuação Total & $\operatorname{Max}=25$ & $\operatorname{Max}=7$ & $\begin{array}{l}\left.{ }^{\star}\right) \text { Em caso de repetição destas palavras, } \\
\text { pontuar apenas UMA vez cada uma. }\end{array}$ & \\
\hline
\end{tabular}




\begin{tabular}{|c|c|c|c|c|}
\hline \multicolumn{5}{|c|}{ F2. SUZANA BORGES } \\
\hline História & $\begin{array}{l}\text { Sinônimos ou ideias } \\
\text { completas }\end{array}$ & $\begin{array}{l}\text { Temas ou ideias } \\
\text { gerais }\end{array}$ & $\begin{array}{l}\text { Sinônimos } \\
\text { (1 ponto) }\end{array}$ & Não pontuar \\
\hline Suzana & & & Suzana, Suzane & Outro nome \\
\hline Borges & & & Borges & Outro sobrenome \\
\hline da periferia & & & Periferia, Subúrbio & Morava \\
\hline de Salvador & & & Salvador, Bahia & Outro local \\
\hline TEMA GERAL (GIST) & & & $\begin{array}{l}\text { INDICACÃ̃O DE QUE } 0 \text { PERSONAGEM PRINCIPAL } \\
\text { ERA MULHER }\end{array}$ & \\
\hline estudante & & & Estudante, Cursava, Estudava, Aluna & \\
\hline de direito & & & Direito, Advocacia & \\
\hline na Universidade & & & Universidade, Faculdade & \\
\hline Federal & & & Federal & \\
\hline TEMA GERAL (GIST) & & & $\begin{array}{l}\text { INDICAÇ̃̃O DE QUE } 0 \text { PERSONAGEM PRINCIPAL } \\
\text { ESTUDAVA }\end{array}$ & \\
\hline reclamou & & & $\begin{array}{l}\text { Reclamou, Denunciou, Expressão da ideia de } \\
\text { relato queixoso. }\end{array}$ & $\begin{array}{l}\text { Disse, Avisou, Declarou, Relatou, } \\
\text { Informou, Fez boletim de } \\
\text { ocorrência }\end{array}$ \\
\hline no escritório & & & Escritório, Sala & Na biblioteca \\
\hline da diretoria & & & Diretoria, Direção, Diretora (*) & Delegacia \\
\hline que tinham sumido & & & $\begin{array}{l}\text { Sumiram, Roubados, Roubaram, Roubo, Foi } \\
\text { roubada, Furto, Expressão da ideia de sumir } \\
\text { sem ser perdido. }\end{array}$ & Havia perdido, Perdeu \\
\hline de cima de sua mesa & & & Mesa, Escrivaninha, Carteira & Dentro da sala de aula \\
\hline na tarde anterior & & & Ultima tarde & Manhã, $2^{\mathrm{a}}$ feira, Aula anterior \\
\hline os livros & & & Livros & \\
\hline que ela tinha comprado & & & Comprado & \\
\hline TEMA GERAL (GIST) & & & $\begin{array}{l}\text { INDICAÇÃO DE QUE O PERSONAGEM DEU FALTA } \\
\text { DE ALGUM PERTENCE }\end{array}$ & \\
\hline Ela disse que ia ter 3 & & & Três & \\
\hline provas & & & Provas, Testes, Exame, Avaliação (*) & \\
\hline TEMA GERAL (GIST) & & & $\begin{array}{l}\text { INDICAÇÃ̃ DE QUE HAVERIA ALGUMA } \\
\text { AVALIAÇÃOO ACADÊMICA }\end{array}$ & \\
\hline $\begin{array}{l}\text { que ainda não tinha } \\
\text { estudado }\end{array}$ & & & Não tinha estudado, Precisava estudar & Não havia recebido \\
\hline $\begin{array}{l}\text { e que os exames } \\
\text { aconteceriam }\end{array}$ & & & Exames $\left({ }^{*}\right)$ & \\
\hline dali a 2 dias & & & Dois & \\
\hline TEMA GERAL (GIST) & & & $\begin{array}{l}\text { INDICAÇÃO DE QUE } 0 \text { PERSONAGEM NÃO } \\
\text { ESTAVA PREPARADO PARA A AVALIAÇÃA }\end{array}$ & \\
\hline A diretora & & & Diretora $\left(^{\star}\right)$ & Universidade, Professora, Chefe \\
\hline $\begin{array}{l}\text { preocupada com a } \\
\text { situação da menina }\end{array}$ & & & Preocupada, Comovida, Sensibilizada, Com dó & Resolveu \\
\hline TEMA GERAL (GIST) & & & $\begin{array}{l}\text { INDICAÇÃO DE QUE A DIRETORA FICOU } \\
\text { SENSIBILIZADA PELA SITUAÇÃO DA } \\
\text { ESTUDANTE }\end{array}$ & \\
\hline emprestou os livros & & & $\begin{array}{l}\text { Emprestou, Forneceu os livros, Emprestou } \\
\text { os livros, Deu os livros, Conseguiu os livros, } \\
\text { Expressão da ideia de providenciar os livros. }\end{array}$ & $\begin{array}{l}\text { Pediu livros emprestados, Pegou } \\
\text { livros para estudar }\end{array}$ \\
\hline para ela. & & & Para ela, Para a menina, Para a estudante & \\
\hline TEMA GERAL (GIST) & & & $\begin{array}{l}\text { INDICACCÃO DE QUE AS NECESSIDADES DA } \\
\text { ESTUDANTE FORAM ATENDIDAS }\end{array}$ & \\
\hline Pontuação Total & $\operatorname{Max}=25$ & $\operatorname{Max}=7$ & 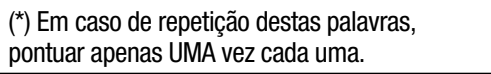 & \\
\hline
\end{tabular}




\begin{tabular}{|c|c|c|c|c|}
\hline \multicolumn{5}{|c|}{ F3. REGINA CARDOSO } \\
\hline História & $\begin{array}{l}\text { Sinônimos ou ideias } \\
\text { completas }\end{array}$ & $\begin{array}{l}\text { Temas ou ideias } \\
\text { gerais }\end{array}$ & $\begin{array}{l}\text { Sinônimos } \\
\text { (1 ponto) }\end{array}$ & Não pontuar \\
\hline Regina & & & Regina & Outro nome \\
\hline Cardoso & & & Cardoso & Outro sobrenome \\
\hline do interior & & & Interior & \\
\hline de Minas gerais & & & Minas Gerais, Minas & Outro local \\
\hline TEMA GERAL (GIST) & & & $\begin{array}{l}\text { INDICAÇ̃̃O DE QUE } 0 \text { PERSONAGEM PRINCIPAL } \\
\text { ERA MULHER }\end{array}$ & \\
\hline instrutora & & & Instrutora, Professora, Dá aula, Ensina & $\begin{array}{l}\text { Funcionaria, Jogadora, Jogava, } \\
\text { Coordenadora, Responsável, } \\
\text { Trabalhava }\end{array}$ \\
\hline de tênis & & & De tênis & \\
\hline no clube & & & Clube & Escola, Empresa \\
\hline da prefeitura & & & Da prefeitura, Do governo & Cidade, Do estado \\
\hline TEMA GERAL (GIST) & & & $\begin{array}{l}\text { INDICAÇÃO DE QUE O PERSONAGEM ERA } \\
\text { ESPORTISTA OU DAVA AULA DE ESPORTE }\end{array}$ & \\
\hline estava brava & & & $\begin{array}{l}\text { Brava, Irritada, Nervosa, Bronqueada, Expressão } \\
\text { da ideia de irritação ou raiva. }\end{array}$ & $\begin{array}{l}\text { Brigaram, Brigou, Discutiu, } \\
\text { Insatisfeita, Chateada }\end{array}$ \\
\hline com os funcionários & & & Funcionários, Empregados & $\begin{array}{l}\text { Aluna(o), Pessoas, Colegas, } \\
\text { Crianças, Meninos, Grupo, } \\
\text { Caboclos, Mulheres, Rapaz }\end{array}$ \\
\hline da limpeza. & & & Limpeza, Faxina & \\
\hline TEMA GERAL (GIST) & & & $\begin{array}{l}\text { INDICAÇÃ0 DE QUE FICOU IRRITADA/BRAVA } \\
\text { COM COLEGAS DO TRABALHO }\end{array}$ & \\
\hline No dia anterior & & & Dia anterior, Expressão da ideia de véspera. & Dia seguinte \\
\hline eles ficaram brincando & & & Brincando, Usaram, Jogando & $\begin{array}{l}\text { Mexeram, Quebraram, Roubaram, } \\
\text { Pegaram }\end{array}$ \\
\hline com as raquetes dela $\mathrm{e}$ & & & Raquetes & Bola \\
\hline $\begin{array}{l}\text { depois não guardaram } \\
\text { de volta }\end{array}$ & & & $\begin{array}{l}\text { Não guardaram, Deixaram bagunçadas, } \\
\text { Deixaram jogadas, Expressão da ideia de não } \\
\text { devolver no lugar. }\end{array}$ & Esconderam, Perderam \\
\hline no lugar certo. & & & Lugar certo, Correto & No armário \\
\hline TEMA GERAL (GIST) & & & $\begin{array}{l}\text { INDICAÇÃO DE QUE OUTROS PEGARAM ALGO } \\
\text { DA PERSONAGEM PRINCIPAL, INDEVIDAMENTE }\end{array}$ & \\
\hline Ela chamou duas & & & Duas, 2 & Pediu ajuda \\
\hline de suas colegas & & & Colega, Amiga, Outra instrutora & Mulheres \\
\hline $\begin{array}{l}\text { para dar uma "lição" } \\
\text { neles. }\end{array}$ & & & $\begin{array}{l}\text { Dar uma lição, Pregar uma peça, Puni-los, } \\
\text { Dar o troco, Se vingar, Expressão da ideia de } \\
\text { descontar. }\end{array}$ & $\begin{array}{l}\text { Ajuda-la, Chamou atenção, } \\
\text { Conversar, Dar um trato }\end{array}$ \\
\hline TEMA GERAL (GIST) & & & $\begin{array}{l}\text { INDICAÇÃO DE QUEA PERSONAGEM PEDIU } \\
\text { AJUDA A ALGUEM PARA SE VINGAR }\end{array}$ & \\
\hline No domingo & & & Domingo & $\begin{array}{l}\text { No dia seguinte, Sábado, No outro } \\
\text { dia, Fim de semana }\end{array}$ \\
\hline elas esconderam & & & Esconderam & Roubaram, Lavar, Pegaram \\
\hline os uniformes deles & & & Uniformes & $\begin{array}{l}\text { Blusa, Camisas, Aventais, } \\
\text { Raquetes, Algo que pertencia, } \\
\text { Roupa }\end{array}$ \\
\hline TEMA GERAL (GIST) & & & $\begin{array}{l}\text { INDICAÇÃO DE QUE O PERSONAGEM FEZ ALGO } \\
\text { CONTRA OS QUEA PREJUDICARAM }\end{array}$ & \\
\hline e só devolveram & & & $\begin{array}{l}\text { Devolveram, Entregaram, Expressão da ideia de } \\
\text { dar de volta. }\end{array}$ & \\
\hline $\begin{array}{l}\text { quando pediram } \\
\text { desculpas }\end{array}$ & & & Desculpas, Expressão da ideia de Perdão. & \\
\hline para ela. & & & Para ela & \\
\hline TEMA GERAL (GIST) & & & $\begin{array}{l}\text { INDICACCÃO DE QUE HOUVE UM PEDIDO DE } \\
\text { DESCULPAS }\end{array}$ & \\
\hline Pontuação Total & $\operatorname{Max}=25$ & $\operatorname{Max}=7$ & & \\
\hline
\end{tabular}




\begin{tabular}{|c|c|c|c|c|}
\hline \multicolumn{5}{|c|}{ M1. LUIS MARQUES } \\
\hline História & $\begin{array}{l}\text { Sinônimos ou ideias } \\
\text { completas }\end{array}$ & $\begin{array}{l}\text { Temas ou ideias } \\
\text { gerais }\end{array}$ & $\begin{array}{l}\text { Sinônimos } \\
\text { (1 ponto) }\end{array}$ & Não pontuar \\
\hline Luis & & & Luis & Outro nome \\
\hline Marques & & & Marques, Marcos & Outro sobrenome \\
\hline TEMA GERAL (GIST) & & & $\begin{array}{l}\text { INDICAÇÃO DE QUE O PERSONAGEM PRINCIPAL } \\
\text { ERA HOMEM }\end{array}$ & \\
\hline adorava & & & Adorava, Gostava muito & Só gostava \\
\hline escutar musica & & & Musica & \\
\hline clássica. & & & Clássica, Erudita & \\
\hline TEMA GERAL (GIST) & & & $\begin{array}{l}\text { INDICAÇÃO DE QUE } 0 \text { PERSONAGEM PRINCIPAL } \\
\text { GOSTAVA DE MÚSICA }\end{array}$ & \\
\hline Seu primeiro & & & Primeiro, Primogênito, Mais velho & \\
\hline filho & & & Filho, Bebê, Filha, Criança, $\left({ }^{\star}\right)$ & \\
\hline nasceu na maternidade & & & $\begin{array}{l}\text { Nasceu na Maternidade, No Hospital, } \\
\text { Nascimento }\end{array}$ & Casa \\
\hline Santa Luzia, & & & Santa Luzia & $\begin{array}{l}\text { Nossa senhora, Outro nome de } \\
\text { santa }\end{array}$ \\
\hline e chorava muito. & & & Chorava Muito, Não parava de chorar, Chorão & \\
\hline TEMA GERAL (GIST) & & & $\begin{array}{l}\text { INDICAÇÃO DE QUE } 0 \text { PERSONAGEM PRINCIPAL } \\
\text { TINHA UMA CRIANÇA }\end{array}$ & \\
\hline Ele percebeu & & & Percebeu, Se deu conta & \\
\hline que 0 silêncio acabara & & & $\begin{array}{l}\text { Silêncio acabara, Casa barulhenta, Fim do } \\
\text { silêncio }\end{array}$ & $\begin{array}{l}\text { Acabou a paz, Acabou paraíso, } \\
\text { Sossego acabou }\end{array}$ \\
\hline $\begin{array}{l}\text { e ele não poderia mais } \\
\text { ouvir }\end{array}$ & & & Ouvir, Escutar & \\
\hline seus CDs & & & CDs, Musicas, Disco & \\
\hline TEMA GERAL (GIST) & & & $\begin{array}{l}\text { INDICAÇÃO DE QUE O PERSONAGEM NÃO } \\
\text { PODIA OUVIR MÚSICAS POR CAUSA DE ALGO } \\
\text { SOBRE A CRIANÇA }\end{array}$ & \\
\hline Quando a criança & & & Criança, Bebê, Filho(a) $\left(^{\star}\right)$ & \\
\hline completou 8 meses & & & 8 meses & 18 meses, 9 meses \\
\hline ele estava desesperado, & & & $\begin{array}{l}\text { Desesperado, Em pânico, Surto, Expressão da } \\
\text { ideia de "extrema alteração de humor". }\end{array}$ & $\begin{array}{l}\text { Estava cansado, Ficou aborrecido, } \\
\text { Indignado, Chateado, Irritado, Não } \\
\text { estava feliz }\end{array}$ \\
\hline TEMA GERAL (GIST) & & & $\begin{array}{l}\text { INDICAÇÃO DE QUE } 0 \text { PERSONAGEM ESTAVA } \\
\text { MUITO INCOMODADO }\end{array}$ & \\
\hline pois o aparelho de som & & & Aparelho de som, Som & $\begin{array}{l}\text { Caixinha de vitrola quebrou, } \\
\text { Pianinho, Radio }\end{array}$ \\
\hline $\begin{array}{l}\text { somente tocava } \\
\text { musiquinhas infantis. }\end{array}$ & & & $\begin{array}{l}\text { Musiquinha ou música infantil, Só ouvia musica } \\
\text { de bebê, Musica infantil, Musica de ninar }\end{array}$ & \\
\hline TEMA GERAL (GIST) & & & $\begin{array}{l}\text { INDICAÇÃO DE QUE OUVIAM MUITA MÚSICA } \\
\text { INFANTIL }\end{array}$ & \\
\hline Então ele trocou o CD & & & Trocou, Expressão da ideia de "colocar outro". & Colocou música, Colocou o cd \\
\hline e colocou uma sinfonia & & & Sinfonia & $\begin{array}{l}\text { Cd, Canção, Colocou a musica, } \\
\text { Música }\end{array}$ \\
\hline de Beethoven. & & & Beethoven & \\
\hline TEMA GERAL (GIST) & & & $\begin{array}{l}\text { INDICAÇÃO DE QUE O PERSONAGEM TROCOU } \\
\text { AS MÚSICAS INFANTIS }\end{array}$ & \\
\hline Para sua surpresa & & & Surpresa, Ficou surpreso, Se espantou & Percebeu \\
\hline a criança se acalmou & & & $\begin{array}{l}\text { Se acalmou, Choro do bebê cessou, Tudo ficou } \\
\text { calmo, Sem barulho, Paz, Tranquila, Silêncio }\left(^{*}\right)\end{array}$ & $\begin{array}{l}\text { Filho gostou, Acordou, Criança } \\
\text { sorriu }\end{array}$ \\
\hline e a casa virou o Paraíso. & & & Palavra Paraíso em qualquer contexto. & \\
\hline TEMA GERAL (GIST) & & & $\begin{array}{l}\text { INDICAÇÃO DE QUE O PERSONAGEM TEVE UM } \\
\text { RESULTADO SATISFATÓRIO }\end{array}$ & \\
\hline Pontuação Total & $\operatorname{Max}=25$ & $\operatorname{Max}=8$ & $\begin{array}{l}\text { (*) Em caso de repetição destas palavras, } \\
\text { pontuar apenas UMA vez cada uma. }\end{array}$ & \\
\hline
\end{tabular}




\begin{tabular}{|c|c|c|c|c|}
\hline \multicolumn{5}{|c|}{ M2. JOSÉ OLIVEIRA } \\
\hline História & $\begin{array}{l}\text { Sinônimos ou ideias } \\
\text { completas }\end{array}$ & $\begin{array}{l}\text { Temas ou ideias } \\
\text { gerais }\end{array}$ & $\begin{array}{l}\text { Sinônimos } \\
\text { (1 ponto) }\end{array}$ & Não pontuar \\
\hline José & & & José, Zé & Outro nome \\
\hline Oliveira & & & Oliveira & Outro sobrenome \\
\hline TEMA GERAL (GIST) & & & $\begin{array}{l}\text { INDICAÇ̃̃O DE QUE } 0 \text { PERSONAGEM PRINCIPAL } \\
\text { ERA HOMEM }\end{array}$ & \\
\hline jogava & & & Jogava, Jogador, Joga & Treinador \\
\hline futebol & & & Futebol, Bola & \\
\hline de salão & & & De salão, Salão & \\
\hline todo domingo & & & Todo domingo, Final de semana & Todo dia, Toda semana \\
\hline na quadra & & & Quadra, Campo & $\begin{array}{l}\text { Centro, Clube, Espaço, Estádio, } \\
\text { Time }\end{array}$ \\
\hline da cooperativa & & & Cooperativa & $\begin{array}{l}\text { União, Escola, Feira, Centro, } \\
\text { Empresa, Time }\end{array}$ \\
\hline agrícola & & & Agrícola, De agricultura, Agrário, Agropecuário & \\
\hline TEMA GERAL (GIST) & & & $\begin{array}{l}\text { INDICAÇÃO DE QUE } 0 \text { PERSONAGEM PRINCIPAL } \\
\text { JOGAVA FUTEBOL }\end{array}$ & \\
\hline $\begin{array}{l}\text { Estava treinando com } \\
\text { o time }\end{array}$ & & & Treinando, No treino, Praticando & $\begin{array}{l}\text { Certo dia, Um dia, Jogo, } \\
\text { Participando }\end{array}$ \\
\hline para um campeonato & & & Campeonato, Torneio & \\
\hline TEMA GERAL (GIST) & & & $\begin{array}{l}\text { INDICAÇÃO DE COMPETIÇÃO, EVENTO } \\
\text { ESPORTIVO }\end{array}$ & \\
\hline quando sua chuteira & & & Chuteira, Tênis, Cadarço, Calçado, Sapato & Chinelo \\
\hline desamarrou. & & & $\begin{array}{l}\text { Desamarrou, Soltou o cadarço, Foi amarrar } \\
\text { [pois estava solto] }\end{array}$ & Amarrar, Esqueceu de amarrar \\
\hline TEMA GERAL (GIST) & & & $\begin{array}{l}\text { INDICAÇÃO DE QUE HOUVE UM PROBLEMA } \\
\text { COM O SAPATO }\end{array}$ & \\
\hline 0 jogador tropeçou & & & $\begin{array}{l}\text { Tropeçou, Escorregou, Expressão da ideia de } \\
\text { algo que o fez cair. }\end{array}$ & Levou um tombo \\
\hline caindo de costas & & & Caindo, Caiu & Tomou carrinho \\
\hline fora do campo. & & & $\begin{array}{l}\text { Fora do campo ou do gramado, Lateral do } \\
\text { campo, Pra fora }\end{array}$ & $\begin{array}{l}\text { Meio do campo, No chão, Quadra, } \\
\text { Estádio }\end{array}$ \\
\hline TEMA GERAL (GIST) & & & $\begin{array}{l}\text { INDICAÇÃO DE QUE } 0 \text { PERSONAGEM PRINCIPAL } \\
\text { CAIU }\end{array}$ & \\
\hline Ele tentou levantar & & & Tentou levantar, Tentou ficar de pé & Se levantou \\
\hline mas sua perna & & & Perna & Fratura em qualquer contexto \\
\hline doía muito. & & & Doía, Sentiu dor & Machucou \\
\hline Foi carregado de maca & & & $\begin{array}{l}\text { Carregado, Levado, Expressão da ideia de que } \\
\text { foi levado por outros. }\end{array}$ & \\
\hline e levado para o vestiário. & & & Vestiário & Hospital, Pronto-socorro \\
\hline TEMA GERAL (GIST) & & & $\begin{array}{l}\text { INDICAÇÃO DE QUE } 0 \text { PERSONAGEM FICOU } \\
\text { MACHUCADO E TEVE QUE SER SOCORRIDO }\end{array}$ & \\
\hline 0 médico & & & Médico, Doutor, Fisioterapeuta & \\
\hline o examinou & & & $\begin{array}{l}\text { Examinou, Diagnosticou, Averiguou, Analisou, } \\
\text { Expressão da ideia de avaliar. }\end{array}$ & Curou \\
\hline TEMA GERAL (GIST) & & & $\begin{array}{l}\text { INDICAÇÃO DE QUE } 0 \text { PERSONAGEM FOI } \\
\text { EXAMINDO POR PROFISSIONAL }\end{array}$ & \\
\hline $\begin{array}{l}\text { e viu que não havia } \\
\text { fratura. }\end{array}$ & & & $\begin{array}{l}\text { Não havia fratura, Não havia quebrado, } 0 \text { osso } \\
\text { estava bem }\end{array}$ & $\begin{array}{l}\text { Não tinha nada, Nada havia } \\
\text { acontecido, Não diagnosticou } \\
\text { nada, não havia ferimento }\end{array}$ \\
\hline $\begin{array}{l}\text { Ele ficou feliz como uma } \\
\text { Criança }\end{array}$ & & & Palavra Criança em qualquer contexto. & $\begin{array}{l}\text { Feliz com a notícia, Ficou feliz, } \\
\text { Ficou muito feliz }\end{array}$ \\
\hline TEMA GERAL (GIST) & & & $\begin{array}{l}\text { INDICAÇÃO DE QUE NÃO HOUVE FERIMENTO } \\
\text { GRAVE }\end{array}$ & \\
\hline Pontuação Total & $\operatorname{Max}=25$ & $\operatorname{Max}=8$ & & \\
\hline
\end{tabular}




\begin{tabular}{|c|c|c|c|c|}
\hline \multicolumn{5}{|c|}{ M3. ALBERTO LEMOS } \\
\hline História & $\begin{array}{l}\text { Sinônimos ou ideias } \\
\text { completas }\end{array}$ & $\begin{array}{l}\text { Temas ou ideias } \\
\text { gerais }\end{array}$ & $\begin{array}{l}\text { Sinônimos } \\
\text { (1 ponto) }\end{array}$ & Não pontuar \\
\hline Alberto & & & Alberto & Outro nome \\
\hline Lemos & & & Lemes, Leme & Outro sobrenome \\
\hline TEMA GERAL (GIST) & & & $\begin{array}{l}\text { INDICAÇ̃̃O DE QUE } 0 \text { PERSONAGEM PRINCIPAL } \\
\text { ERA HOMEM }\end{array}$ & \\
\hline do interior & & & Interior & \\
\hline de Goiás & & & Goiás & Outro local \\
\hline plantava & & & $\begin{array}{l}\text { Agricultor, Plantação, Produtor, Plantador, } \\
\text { Cultivava, Colhia }\end{array}$ & Fazendeiro \\
\hline verduras e & & & Verduras & Alface, Frutas, Limões, Frutos \\
\hline legumes & & & Legumes & $\begin{array}{l}\text { Chuchu, Feijão,Batata, Milho, } \\
\text { Vegetais }\end{array}$ \\
\hline no seu sítio. & & & Sitio, Fazenda, Chácara & Horta \\
\hline TEMA GERAL (GIST) & & & $\begin{array}{l}\text { INDICAÇ̃̃̃ DE QUE } 0 \text { PERSONAGEM } \\
\text { TRABALHAVA COM A TERRA }\end{array}$ & \\
\hline Toda semana, & & & Toda semana & Todo fim de semana, Todo dia \\
\hline ele ia vender os produtos & & & Vender os produtos, Venda, Comercializava & Fazia entrega, Comprava \\
\hline em Itapira. & & & Itapira & Outra cidade \\
\hline TEMA GERAL (GIST) & & & $\begin{array}{l}\text { INDICAÇÃO DE QUE } 0 \text { PERSONAGEM PRINCIPAL } \\
\text { VENDIA } 0 \text { QUE PRODUZIA OU PLANTAVA }\end{array}$ & \\
\hline 0 verão & & & Verão, A estação & 0 tempo, Clima \\
\hline estava escaldante, & & & $\begin{array}{l}\text { Escaldante, Calor, Muito sol, Sol forte, Quente, } \\
\text { Ensolarado, Quente, Muito quente, Expressão } \\
\text { da ideia de muito calor. }\end{array}$ & $\begin{array}{l}\text { Estiagem, Terra árida, Verduras } \\
\text { secas }\end{array}$ \\
\hline TEMA GERAL (GIST) & & & $\begin{array}{l}\text { INDICAÇÃO DE QUE } 0 \text { TEMPO ESTAVA MUITO } \\
\text { QUENTE }\end{array}$ & \\
\hline 0 ar estava seco, & & & $\begin{array}{l}\text { Ar seco, Seca, Tempo seco, Árido, Clima árido, } \\
\text { Expressão da ideia de secura. }\end{array}$ & Ar rarefeito, Mal tempo \\
\hline $\begin{array}{l}\text { e não havia previsão de } \\
\text { chuva }\end{array}$ & & & $\begin{array}{l}\text { Sem previsão de chuva, Escassez de chuva, } \\
\text { Falta de chuva, Chuva não vinha }\end{array}$ & Falta de água, Acabou água \\
\hline para aquela região. & & & Região, Área, Redondeza & \\
\hline TEMA GERAL (GIST) & & & $\begin{array}{l}\text { INDICAÇÃO DE QUE ESTAVA SECO OU SEM } \\
\text { CHUVA }\end{array}$ & \\
\hline Então ele teve medo & & & Medo, Preocupação, Ansioso & Achou, Pensou \\
\hline que a colheita & & & Colheita, Safra & $\begin{array}{l}\text { Perder tudo, Que a plantação } \\
\text { suasse, Não nascesse, Safra dar } \\
\text { errado, Perder a plantação }\end{array}$ \\
\hline fosse prejudicada. & & & Prejudicada, Afetada & Não teria êxito \\
\hline TEMA GERAL (GIST) & & & $\begin{array}{l}\text { INDICAÇ̃̃O DE QUE } 0 \text { PERSONAGEM PRINCIPAL } \\
\text { ESTAVA PREOCUPADO }\end{array}$ & \\
\hline $\begin{array}{l}\text { Como o tempo não } \\
\text { mudaria, }\end{array}$ & & & Tempo não mudaria, Não ia virar o tempo & Tempo ruim \\
\hline $\begin{array}{l}\text { ele resolveu tomar uma } \\
\text { providência. }\end{array}$ & & & $\begin{array}{l}\text { Resolveu tomar providencia, Decidiu agir, } \\
\text { Expressão da ideia de resolver agir. }\end{array}$ & Teve ideia \\
\hline Contratou & & & Contratou, Alugou, Pagou & Comprar, Usa, Ajuda \\
\hline caminhões-pipa & & & Caminhão-pipa, Caminhão de água & Alguém, Pipa \\
\hline TEMA GERAL (GIST) & & & $\begin{array}{l}\text { INDICAÇÃO DE QUE O PERSONAGEM FEZ ALGO } \\
\text { SOBRE A FALTA DE ÁGUA }\end{array}$ & \\
\hline para regar a plantação & & & $\begin{array}{l}\text { Regar, Pulverizar, Molhar, Fornecer água, } \\
\text { Molhar, Aguar, Expressão da ideia de regar. }\end{array}$ & $\begin{array}{l}\text { Cuidar da lavoura, Cultivar, } \\
\text { Transportar }\end{array}$ \\
\hline $\begin{array}{l}\text { que ficou verde como } \\
\text { Esmeralda. }\end{array}$ & & & Palavra Esmeralda em qualquer contexto. & $\begin{array}{l}\text { Linda e verde, Mais verde, Ficou } \\
\text { verde, Verde e brilhante }\end{array}$ \\
\hline TEMA GERAL (GIST) & & & $\begin{array}{l}\text { INDICACĈ̃O DE QUE FOI RESOLVIDO } 0 \\
\text { PROBLEMA DE FALTA DE CHUVA OU ÁGUA }\end{array}$ & \\
\hline Pontuação Total & $\operatorname{Max}=25$ & $\operatorname{Max}=8$ & & \\
\hline
\end{tabular}

